\title{
Kepler eclipsing binaries with $\delta$ Scuti components and tidally induced heartbeat stars
}

\author{
Zhao Guo, Douglas R. Gies and Rachel A. Matson \\ Center for High Angular Resolution Astronomy and Department of Physics and Astronomy, \\ Georgia State University, P. O. Box 5060, Atlanta, GA 30302-5060, USA \\ email: guo@astro.gsu.edu, gies@astro.gsu.edu, rmatson@astro.gsu.edu
}

\begin{abstract}
Scuti stars are generally fast rotators and their pulsations are not in the asymptotic regime, so the interpretation of their pulsation spectra is a very difficult task. Binary stars, especially eclipsing systems, offer us the opportunity to constrain the space of fundamental stellar parameters. Firstly, we show the results of KIC9851944 and KIC4851217 as two case studies. We found the signature of the large frequency separation in the pulsational spectrum of both stars. The observed mean stellar density and the large frequency separation obey the linear relation in the log-log space as found by Suarez et al. (2014) and García Hernández et al. (2015). Second, we apply the simple 'one-layer model' of Moreno \& Koenigsberger (1999) to the prototype heartbeat star KOI-54. The model naturally reproduces the tidally induced high frequency oscillations and their frequencies are very close to the observed frequency at 90 and 91 times the orbital frequency.
\end{abstract}

Keywords. (Stars:) binaries: eclipsing, Stars: oscillations, Techniques: spectroscopic

\section{Introduction}

The standard procedure of analyzing eclipsing binaries (EBs) is to combine the photometry with spectroscopy to determine the accurate fundamental stellar parameters like mass, and radius. Then we can compare with stellar evolution models and derive other physical parameters like ages, core overshooting parameter etc.

We started a spectroscopic follow up of 20 Kepler EBs which show $\delta$ Scuti type pulsations (Gies et al. 2012). $\delta$ Scuti variables are A- or F-type stars; they are p-mode pulsators with pulsation periods from $15 \mathrm{~min}$ to several hours. Here we show the results of two case studies.

\section{KIC9851944 and KIC4851217}

KIC 9851944 is a short period $(P=2.16$ days $)$ eclipsing binary in the Kepler field of view. By combining the analysis of Kepler photometry and phase resolved spectra from Kitt Peak National Observatory and Lowell Observatory, we determine the atmosphere parameters and fundamental parameters of both stars. The two components have very different radii $\left(2.27 R_{\odot}, 3.19 R_{\odot}\right)$ but close masses $\left(1.76 M_{\odot}, 1.79 M_{\odot}\right)$ and effective temperatures $(7026 \mathrm{~K}, 6902 \mathrm{~K})$, indicating different evolutionary stages. The primary is still on the main sequence (MS), while the larger secondary star has evolved to post-MS, burning hydrogen in a shell. A comparison with coeval evolutionary models suggests that the stars have solar metallicity and that a higher mass ratio is required to fit the radii and temperatures of both stars simultaneously. Both components show $\delta$ Scuti type pulsations which we interpret as p-modes and $\mathrm{p}$ and $\mathrm{g}$ mixed modes. 


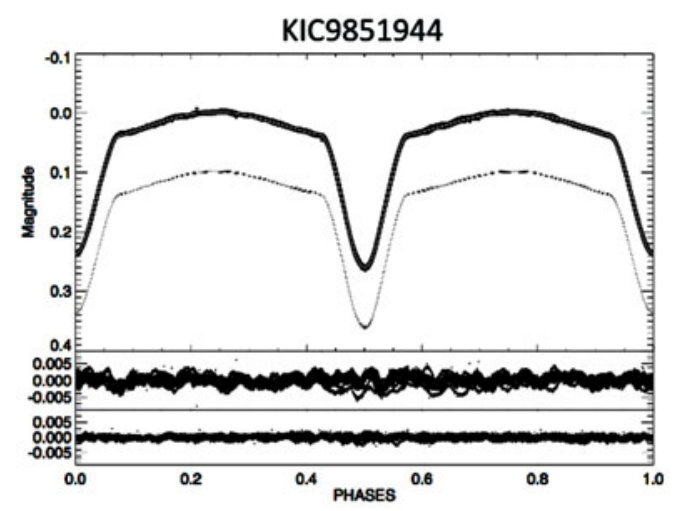

KIC4851217

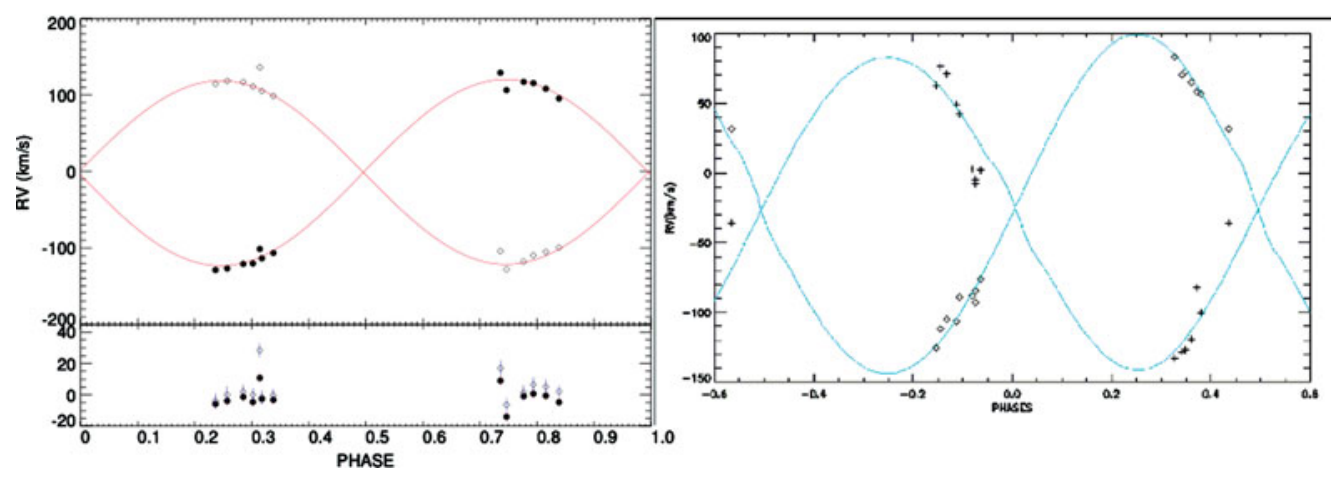

Figure 1. The light curve and radial velocity curve of KIC9851944 and KIC4851217. The best fitting models are from the ELC code. The results for KIC4851217 are preliminary, so no residual plots are shown.

KIC4851217 is an eccentric system with $e \approx 0.04$. The preliminary results show that the two components have masses of $1.91 M_{\odot}$ and $1.76 M_{\odot}$, radii of $2.81 R_{\odot}$ and $2.69 R_{\odot}$.

The light curve and radial velocity curve and the corresponding best fitting models of both systems are shown in Figure 1.

For both systems, we found a regular frequency pattern corresponding to the large frequency separation in the power spectrum of the detected frequencies. For KIC9851944, the observed large frequency separation agrees with the density of the secondary star. For KIC4851217, the two components have similar densities and they are both consistent with the observed large separation (Fig. 2). The observed frequency regularity and the mean stellar density follow closely the $\Delta \nu-\bar{\rho}$ relation of $\delta$ Scuti stars from Suarez et al. (2014) and García Hernández et al. (2015) as shown in Figure 3.

\section{KOI-54: Application of the One-Layer Model}

The simple 'one-layer model' proposed in Moreno \& Koenigsberger (1999) has been applied to the study of dynamical tidal flow in eccentric binaries. In this model, the star is composed of two parts: the inner body with uniform rotation and the surface layer, which is subject to tidal perturbation from the companion. The stellar surface consists of concentric rings. The equation of motion of each brick element in the rings is solved numerically. The motion is calculated from first principle and takes into account of the gravitational force, centrifugal force, Coriolis force, gas pressure, and viscous force. 


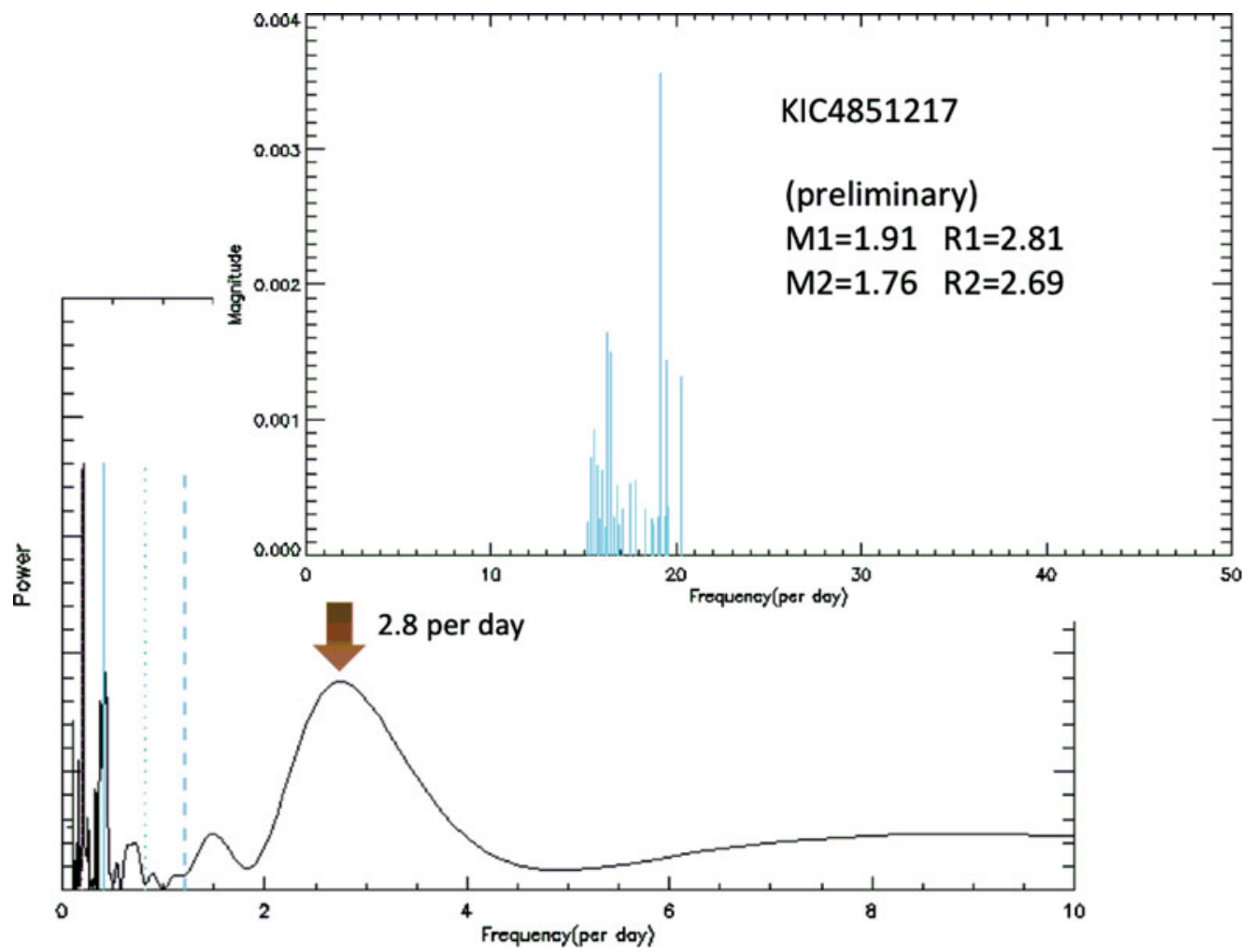

Figure 2. The pulsation spectrum of KIC4851217 and the frequency regularity at $2.8 d^{-1}$ found in its Fourier transform

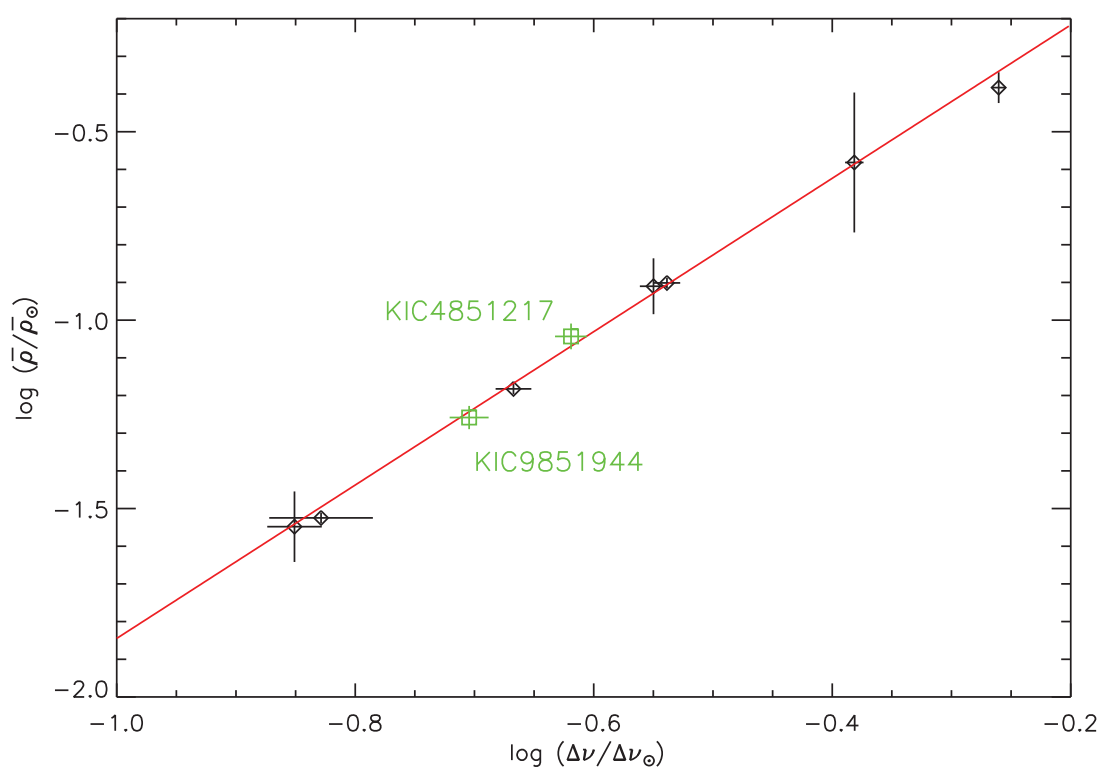

Figure 3. The relation between the observed mean stellar density and the observed frequency regularity in KIC9851944 and KIC4851217 (green squares). The diamonds represent for six $\delta$ Scuti pulsating EBs and a single $\delta$ Scuti star with a interferometrically determined radius (Rasalhague) from García Hernández et al. (2015). 


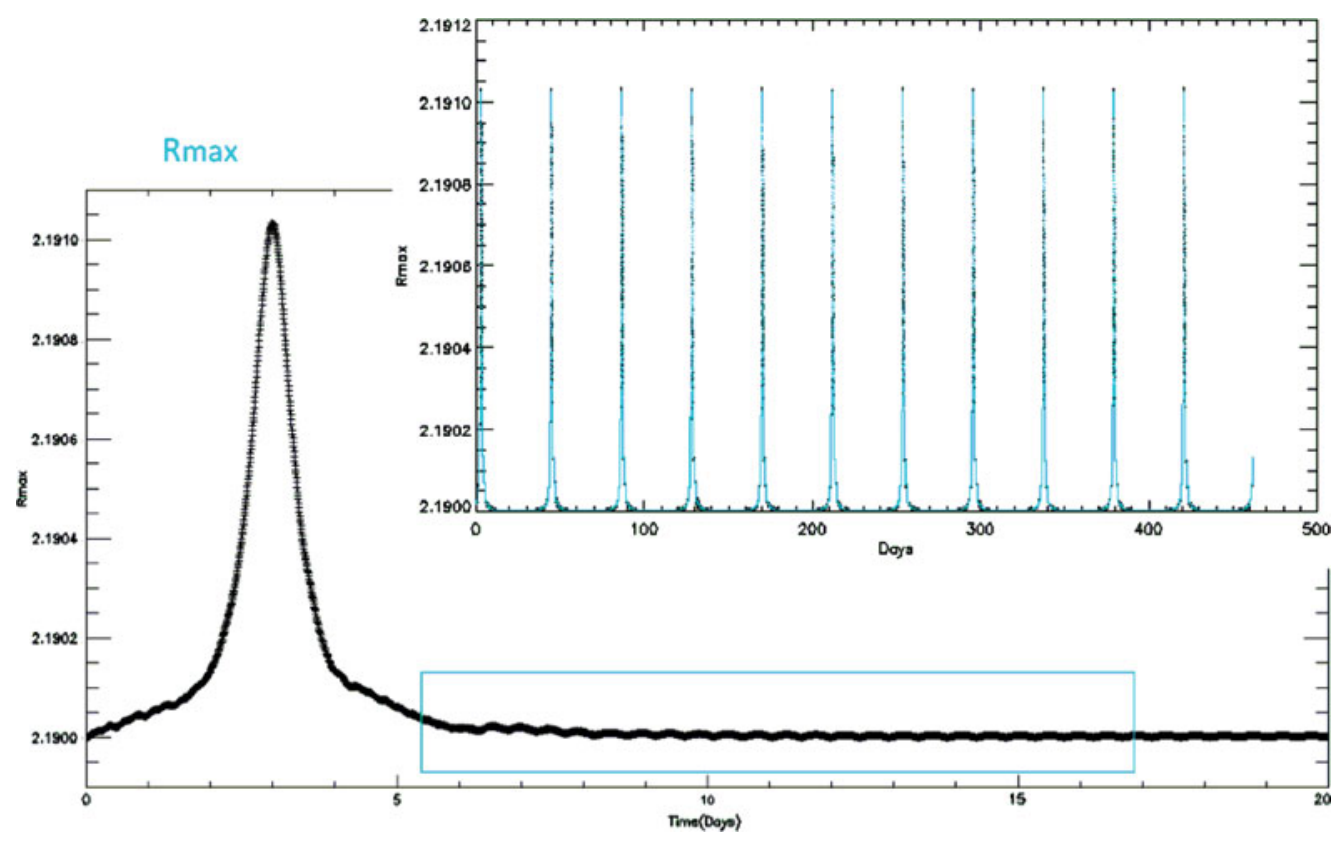

Figure 4. The variations of $R_{\max }$ (the maximum radius on the equator) from the first periastron passage to 450 days later, calculated from the one-layer model using the parameters of KOI-54. The peaks correspond to periastron passages. The tidally induced oscillations shown in the lower rectangle are obvious after the periastron passage.

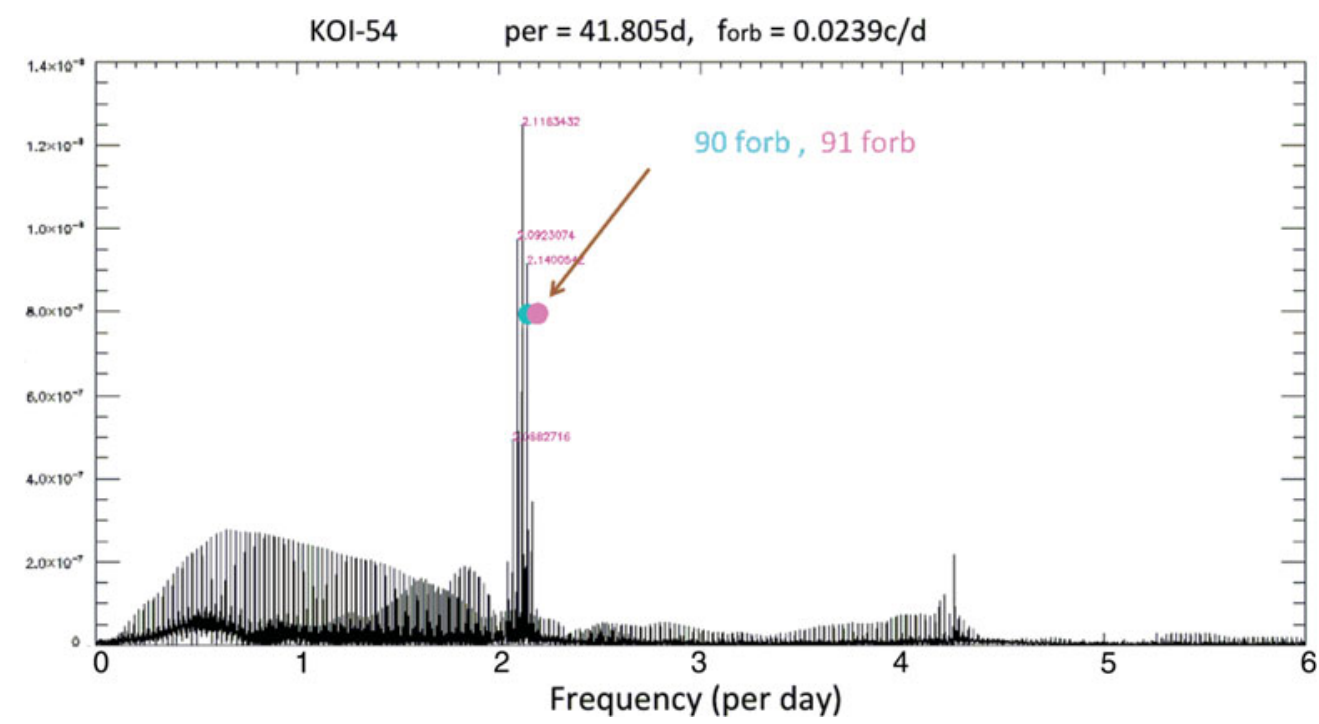

Figure 5. The Fourier spectrum of the variations of $R_{\max }$ (the maximum radius on the equator) from the one-layer model using the parameters of KOI-54. The observed tidally induced pulsations at 90 and 91 times of orbital frequency are indicated by the blue and pink dots.

The model has successfully explained the observed line profile variations in $\epsilon$ Per (Moreno et al. 2005) and Spica (Harrington et al. 2009; Palate et al. 2013). The model can also reproduced the synchronization timescale for binaries (Toledano et al.2007; Moreno et al. 2011). 
We use the parameters of KOI-54 from Welsh et al. (2011). The one-layer model outputs the distorted stellar surface at each phase of the binary orbit. The maximum radius on the stellar equator has an obvious high frequency oscillation except around the periastron when the star is elongated by the equilibrium tide (Figure 4). The frequency of this oscillation is very close to the observed tidally induced oscillation at 90 and 91 times the orbital frequency as shown in Figure 5 . This is also consistent with the interpretation that the dominant tidally induced oscillation is a $l=2, m=0$ mode as suggested by O'Leary \& Burkart (2014) and Fuller \& Lai (2012). It is worthwhile to investigating whether this simple model can reproduce the tidally induced oscillations in other heartbeat stars.

\section{Future Work}

We find many other Kepler $\delta$ Scuti EBs with frequency regularities, e.g., KIC 8262223 and KIC 8553788 in our sample. We also have interferometric observations from CHARA Array of two well-studied $\delta$ Scuti stars 44 Tau and 29 Cygni. Deriving accurate radii for both stars will be a great help in interpreting the pulsation spectra of these $\delta$ Scuti stars.

\section{Acknowledgments}

We thank Koenigsberger Gloria and Moreno Edmundo for making the TIDES code available to us. We thank García Hernández for useful discussions. We acknowledge the observations taken using the 4-m Mayall telescope at the KPNO and Perkins telescope Lowell Observatory. This work is partly based on data from the Kepler mission, which is funded by the NASA Science Mission directorate. The photometric data were obtained from the Mikulski Archive for Space Telescopes (MAST). This study was supported by NASA grants NNX12AC81G, NNX13AC21G, and NNX13AC20G.

\section{References}

Fuller, J. \& Lai, D. 2012, MNRAS, 420, 3126

García Hernández, A., Martín-Ruiz, S., Monteiro, M. J. P. F. G., et al. 2015, ApJL, in press (arXiv:1509.01111)

Gies, D. R., Williams, S. J., Matson, R. A., et al. 2012, AJ, 143, 137

Harrington, D., Koenigsberger, G., Moreno, E., \& Kuhn, J. 2009, ApJ, 704, 813

Moreno, E. \& Koenigsberger, G. 1999, RMxAA, 35, 157

Moreno, E., Koenigsberger, G., \& Toledano, O. 2005, A\&A, 437, 641

Moreno, E., Koenigsberger, G., \& Harrington, D. M. 2011, A\&A, 528, A48

O'Leary, R. M. \& Burkart, J. 2014, MNRAS, 440, 3036

Palate, M., Koenigsberger, G., Rauw, G., Harrington, D., \& Moreno, E. 2013, A\& A, 556, A49

Suárez, J. C., Garcia Hernández, A., Moya, A., et al. 2014, A\&A, 563, 7

Toledano, O., Moreno, E., Koenigsberger, G., Detmers, R., \& Langer, N. 2007, A\&A, 461, 1057

Welsh, W. F., Orosz, J. A., Aerts, C., et al.2011, ApJS, 197, 4

\section{Discussion}

FUller AND PRSA: Is the result of KOI-54 from the one-layer model sensitive to model parameters?

GuO: Not very. The tidally induced oscillation can vary from 80 to 100 times the orbital frequency if we vary the model parameters like the viscosity parameter, the layer depth, and spin frequency, for example. 